Cahiers de recherches médiévales

\title{
Bon et mauvais langage :la parole multipliée chez Christine de Pizan
}

\section{Liliane Dulac}

\section{(2) OpenEdition \\ 1 Journals}

Édition électronique

URL : https://journals.openedition.org/crm/934

DOI : 10.4000/crm.934

ISSN : 1955-2424

Éditeur

Honoré Champion

Édition imprimée

Date de publication : 15 février 1999

ISSN : $1272-9752$

Référence électronique

Liliane Dulac, «Bon et mauvais langage :la parole multipliée chez Christine de Pizan », Cahiers de recherches médiévales [En ligne], 6| 1999, mis en ligne le 11 janvier 2007, consulté le 15 décembre 2022. URL : http://journals.openedition.org/crm/934 ; DOI : https://doi.org/10.4000/crm.934

Ce document a été généré automatiquement le 15 décembre 2022.

Tous droits réservés 


\title{
Bon et mauvais langage :la parole multipliée chez Christine de Pizan
}

\author{
Liliane Dulac
}

1 D'un bout à l'autre de l'œuvre de Christine de Pizan court un thème très divers dans sa forme et sa portée, mais toujours aisément identifiable : celui de la parole qui vole de bouche en bouche, s'amplifiant à mesure qu'elle se propage, non sans entraîner les plus grandes conséquences ${ }^{1}$. Le plus souvent dangereuse, elle est, avant de devenir collective et anonyme, l'arme des médisants qui perdent les amants imprudents, des serviteurs qui diffament leurs maîtres, des séditieux qui soulèvent le peuple, et de bien d'autres acteurs d'une dramaturgie verbale qui semble parfois se confondre avec la vie sociale, notamment dans les cours. Mais il existe également une parole bénéfique, qui tout aussi capable de se propager et de se fortifier, résume en elle les plus hautes valeurs morales; cette bonne renommée, comme le «mauvais langage », peut elle aussi être une arme, au moins défensive, et un instrument politique.

Dans ses différentes variantes, le thème est caractérisé par d'assez visibles constantes, qui tiennent au lexique, aux images et à certains schémas narratifs. Car cette parole qui ne naît que pour s'étendre ne se conçoit qu'en mouvement, ou plutôt en action : elle n'est jamais sans effets. Dans l'œuvre de Christine, elle est évoquée sur deux registres principaux, apparemment éloignés, au moins au départ: celui de la poésie amoureuse issue de la tradition courtoise, où le topos des «losengiers " ${ }^{2}$ est des plus rebattus; et d'autre part, comme objet d'une réflexion morale, puis politique, très concrètement fondée sur l'analyse de certaines réalités contemporaines. Entre ces deux registres, on n'observe pas de séparation absolue, mais plutôt de l'un à l'autre, certains glissements, et surtout un enrichissement considérable. C'est précisément cette métamorphose et cette extension remarquable du champ thématique qui nous paraissent mériter examen : pratiquer à cet endroit une sorte de coupe diachronique ne devrait manquer pas d'apporter quelques lumières sur la dynamique qui emporte l'œuvre, à mesure que croissent ses ambitions.

3 Aucun mot ne peut désigner la parole colportée et "semée ${ }^{3}$ en rendant compte de l'ensemble des modalités qu'elle revêt dans les écrits de Christine; ni aucun mythe, bien que celui de Fama, auquel elle donne une si grande place dans la première partie de 
l'Advision Cristine $e^{4}$ à propos de la situation du royaume, symbolise certaines de ses formes d'existence les plus importantes. Notons déjà à ce sujet, car nous ne pourrons nous $\mathrm{y}$ attarder, que le thème que nous nous proposons d'étudier a aussi beaucoup de rapport avec un autre mythe allégorique, central dans l'Advision, celui d'Opinion: de celle-ci partent en effet des discours incertains et souvent dangereux, qui se multiplient et s'étendent d'autant plus qu'ils se contredisent. Pour nous en tenir à un préliminaire plus modeste, mais délicat, passons rapidement en revue le lexique, qui est riche et relativement homogène : c'est un fait très notable et significatif que du premier recueil de ballades constitué par Christine jusqu'à ses traités moraux et politiques, les mêmes mots sont généralement employés pour désigner les actes de langage, le plus souvent coupables, qui sont à l'origine de la parole anonyme et collective. Ainsi, outre "dire» et «mesdire», on rencontre fréquemment «blasmer", «jengler", "faire rapport», "diffamer», «compter», "plait semer $»^{5} . .$. À côté de «paroles» (souvent «mauvaises»), «voix ${ }^{6}$, «cry» (au sens de proclamation), "diffame» et "vilain langage», on trouve par exemple associés, pour paraphraser une sentence de saint Augustin ${ }^{7}$, "faulse accusation», "murmuracion», "detraccion», " faulx rapport », « diffamacion », tous péchés de langue propres à faire naître la rumeur défavorable. «Renommee» peut avoir un sens voisin : rappelant qu'on a médit de ses mœurs, Christine évoque les « renommees » qui ont circulé à son propos. On rencontre plus rarement «rumeur" au sens de bruit malveillant (Prod'ommie de l'omme) $)^{8}$, car le terme désigne plutôt la révolte ou le mouvement de paroles qui la précède immédiatement ; «murmure», beaucoup plus fréquent, a les deux valeurs : Christine prévoit dans la Cité des Dames " "que maintes murmures naistront entre les mesdisans de ceste present oeuvre ", mais dans ses derniers traités politiques, elle usera du mot pour désigner la parole menaçante du populaire insurgé. Ceux qui sont à l'origine du «mauvais langage» sont abondamment qualifiés, comme tel chevalier «mal parlier, jengleur, plein de mesdis $»^{10}$, parmi d'autres qui sont «venteurs», «mesdiseurs», «deceveurs». Le vocabulaire spécifique de la parole favorable est lui beaucoup plus réduit, d'autant plus que les termes qui la désignent ordinairement peuvent, comme "renommee», "renom», "fame», avoir également un sens péjoratif ${ }^{11}$. Si dans ce lexique certaines valeurs tendent progressivement à prendre plus de poids, notamment lorsque les aspects politiques l'emportent, on ne perçoit pas de rupture ni de cloisonnement entre les différents domaines où se manifestent les effets de la parole collective $^{12}$.

4 Afin de faciliter l'étude des transformations qui affectent la représentation de ce phénomène chez Christine, et sans dissimuler la part d'arbitraire que comporte cette décision, nous distinguerons deux grands massifs qu'aucune limite chronologique précise ne sépare: d'une part les œuvres poétiques ${ }^{13}$ ainsi que les débats sur les matières d'amour et sur le Roman de la Rose ${ }^{14}$ de Jean de Meung, qui dans l'ensemble correspondent à la première partie de sa carrière littéraire (bien que le recueil des Cent balades d'amant et de dame soit beaucoup plus tardif) ; de l'autre, des œuvres didactiques en vers et en prose, traités moraux et politiques et aussi écrits de conseil et d'enseignement inspirés par les circonstances, comme la Lamentacion sur les maux de la France ou l'Epistre à la Reine.

5 Dans les premières poésies de Christine, le thème de la "rumeur " s'identifie presque entièrement à celui des médisants qui guettent les amants et déshonorent les dames par les propos qu'ils colportent. Cette menace confuse est un élément indispensable de 
la dramaturgie amoureuse : le plus souvent, qu'elle soit effective, redoutée ou peut-être simplement supposée, la parole des médisants n'est guère considérée en elle-même, mais plutôt comme un obstacle convenu opposé à la relation des amants. Elle joue les utilités en offrant au meneur du jeu poétique les ressources d'une gamme assez étendue de discours, selon les situations et les points de vue. Le caractère conventionnel de ce dispositif est confirmé par le peu d'attention porté à la bonne renommée, qui n'est guère évoquée que dans la mesure où elle sert d'enjeu, compromise par les uns ou sauvée par les autres, les bons chevaliers champions de l'honneur des dames, comme Charles d'Albret dont Christine fait l'éloge en lui promettant à lui-même "grant renommée » (Autres balades, II, v. $12^{15}$ ). On peut remarquer également que dans le cas le plus courant la parole des médisants, telle qu'elle est représentée, n'a qu'une existence floue, ce qui est un des caractères de ce que nous appelons rumeur ${ }^{16}$ : au point qu'il est même possible de jouer de son inconsistance, comme il arrive dans les Cent balades d'amant et de dame, lorsque l'amant se met soudain à invoquer de manière répétée la crainte qu'il a du «murmure/ De mesdisans» pour expliquer son absence (ballade LXXXIX, vv. 5-6 ${ }^{17}$ ) : sa dame a toute raison de ne voir qu'un faux-fuyant dans ce qui n'est guère que la rumeur d'une rumeur, ou un simple mensonge.

On ne doit cependant pas méconnaitre que Christine parvient à découvrir dans cette thématique rebattue quelques ressources nouvelles qui s'avèreront fécondes. En fait, elle semble avoir très tôt porté une attention précise à quelques-uns de ses aspects qui ne sont pas sans rapport avec une certaine réalité observable dans la société de cour. De cet intérêt, on peut trouver un premier indice dans quelques poèmes où sans innover réellement par rapport aux conventions du genre, Christine donne un tour particulièrement vif à la dénonciation de personnages traités comme des types, bien caractérisés par leur langage, leurs attitudes et leurs actions : à côté du chevalier médisant que nous avons déjà rencontré, amateur de virelais et de «beaulz ditz », mais qu'on ne dit «vaillant » que par antiphrase, elle met en scène d'autres figures de même farine :

Grans vanteurs sont et sanz proece,

Mais trés bien parez par dehors,

Orgueilleux pour leur gentillece

Et tiennent bien aise leurs corps ;

[...]

Il n'est nulle si grant maistrece,

Ne femme autre, soit droit ou tors,

Que leur fausse lengue ne blece

Leur bon renom; aise sont lors

Quant ilz en font mauvais rapors,

Qui s'i vouldra mirer s'y mire ;

Mais mieulx que vifs vaulsissent mors,

N'on n'en pourroit assez mesdire

(Autres Balades XVII, vv. 9-12; 17-2418)

7 Le poème se fait donc miroir, en caractérisant des personnages supposés reconnaissables, et si ce tour satirique relève également de la tradition, on peut néanmoins y voir le départ d'une veine réaliste à visée morale. À la parole néfaste de ces gens «plus que pors vilz et ors» (ibidem, v. 7), répond dès lors cette autre parole qui elle aussi circule et peut se multiplier, celle du poète, lui aussi faiseur de renommée. C'est ainsi qu'à la ballade que nous venons de citer, fait écho la conclusion d'un virelai : 
Telle gent sont drois villains purement,

Et devroit on leur renom amortir,

Se pris et los estoit a departir

(Virelays XII, vv. 20-22 ${ }^{19}$ )

8 Ce type de portraits est repris et développé dans l'Epistre au dieu d'amour. Certains constituent de véritables petits tableaux de mœurs, où sont longuement évoquées les assemblées de "compaings", qui dans les tavernes, à la cour des ducs ou chez le roi, échangent joyeusement des confidences sur leurs amours, malgré d'hypocrites réticences. Quel qu'en soit le mobile, vanité, dépit ou simple entraînement entre camarades, des femmes en font inévitablement les frais, car le thème de ces conversations est présenté comme habituel et stéréotypé :

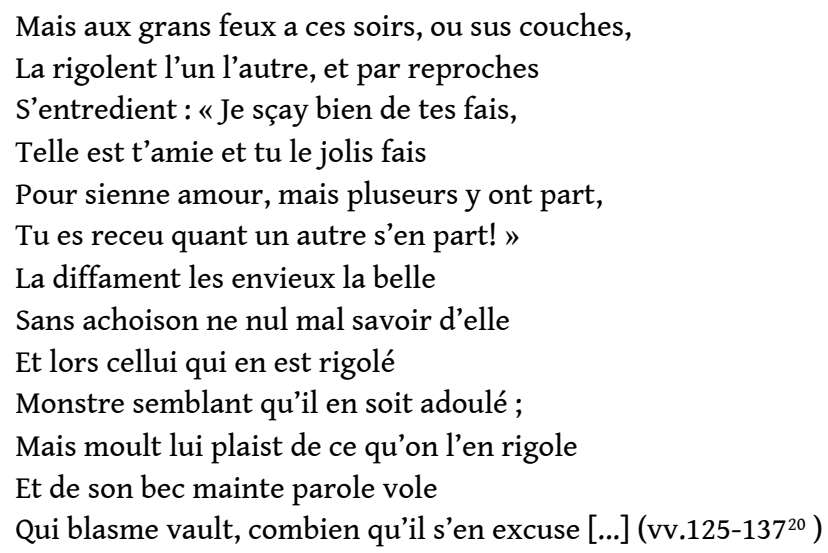

9 De telles scènes, où la médisance exercée aux dépens d'une femme ou d'une autre, prend la forme d'un jeu viril, sont représentées avec assez de détail pour prendre valeur d'analyse d'un comportement collectif: l'enchaînement ordinaire des propos exprime et conforte à la fois le mépris qui atteint les femmes dans leur ensemble. Cette généralisation est déterminée par les mœurs masculines de la cour, si bien que par une sorte de métonymie, on passe de la médisance particulière aux propos qui abaissent toutes les femmes, puis aux écrits qui mettent en forme le même fond de lieux communs :

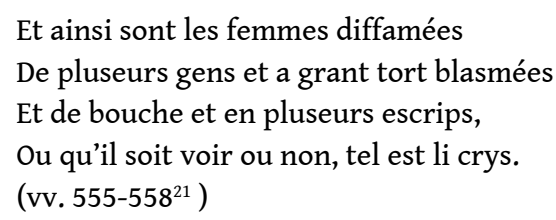

10 En partant d'une représentation précise et colorée des pratiques masculines de la médisance, Christine en vient ainsi à faire apparaître les ouvrages dirigés contre les femmes comme un prolongement de la rumeur confuse qui trouve son origine dans des comportements grossiers et vils, voire dans des propos de taverne.

11 L'observation des mœurs qui dans l'Epistre au dieu d'amour fournit le point de départ d'une réfutation argumentée des thèses misogynes, se prolonge ailleurs en conseils à l'adresse des femmes. Cette instruction, qui fait pour l'essentiel référence à la vie de cour, prend elle aussi pour base une analyse des cheminements divers de la médisance et de ses conséquences. Il s'agit d'une morale de précaution, esquissée par exemple dans la pièce XLIII des Autres ballades dont les strophes évoquent successivement la nécessité d'éviter "le contraire/De bon renom», le danger des "parolles semées/En divers lieux » que suscite la coquetterie, enfin les trompeurs qui médisent «sans cause » 
des femmes à qui ils ont tenté de plaire. De façon beaucoup plus détaillée, une leçon identique est exposée dans le Livre du Duc des vrais amans ${ }^{22}$, sous la forme de la lettre que la dame Sebille de la Tour adresse à la duchesse dont elle a été la gouvernante. De ce morceau bien connu, retenons seulement qu'il est lui aussi construit comme une présentation des multiples dangers que la rumeur fait courir à une femme de haut rang: alertée par les bruits qui courent sur la conduite de sa protégée, la dame de la Tour lui rappelle les écueils où peut se perdre sa «renommee», comme celle de toute femme qui se laisse prendre au piège de l'amour courtois. Ce faisant, elle insiste particulièrement sur certaines constantes qui gouvernent le monde de la rumeur. Quelques attitudes imprudentes suffisent à la faire naître, aux dépens de la dame même innocente, et elle circule et grossit dès lors d'elle même :

[...] et pour un petit de nyce semblant, par aventure fait par joennesce et sans malice, les mauvaises langues jugeront et y adjousteront de choses qui oncques ne furent faittes ne pensées, et ainsi va tel language de bouche en bouche qui n'est mie apetissiez, ainz tousjours acreu. (t. III. p. 165).

Quant au service amoureux de l'amant courtois, après avoir conduit la dame trop crédule à «mettre son corps et son honneur ou dongier des langues et es mains de telz servans ", il ne s'achève pas sans la livrer à de plus grands périls :

[...] et en la fin de telle amour souventes fois le blasme et parler des gens aux dames en demeure, ou a tout le moins la craintte et paour en leurs cuers que ceulx mesmes en qui se sont fiées le dient et s'en vantent ou aulcun aultre qui le tour sache.[...] (t. III, p. 169)

On sait que cette page qui développe et systématise l'analyse que proposaient notamment l'Epistre au dieu d'amour et d'autres poèmes, sera reprise quelques années plus tard dans le Livre des trois Vertus ${ }^{23}$ pour appuyer l'instruction adressée aux princesses et aux grandes dames. Christine a donc poursuivi sur cette question une réflexion qui n'a cessé de s'enrichir et de se préciser. En attendant de considérer de plus près le traitement très élaboré que la question recevra dans ce traité, notons que cet approfondissement continu suggère fortement que le thème de la rumeur médisante, bien loin de n'être que de convention, comme on pourrait en juger d'après les premiers poèmes, tend à rendre compte avec une efficacité croissante d'une réalité de première importance dans la vie de cour: la rumeur y est un phénomène d'autant plus difficile à maîtriser qu'il est dans une large mesure indépendant des caractères et des situations individuels, puisque déterminé par des habitudes sociales et une mentalité collective.

Cette recherche qu'on peut suivre dans l'œuvre de Christine au cours des années qui précèdent ses grands traités en prose concerne pour l'essentiel la condition des femmes de la cour. Mais il lui arrive de manifester son intérêt pour d'autres formes de la parole colportée, d'une portée plus générale : par exemple quand elle évoque les médisants qui contrefont la vertu pour mieux s'en prendre à chacun (une forme d'hypocrisie que Gerson dénonce dans un de ses traités ${ }^{24}$ ):

Simples et bons semblent de premier bont, Mais en y a qui sont de Judas pire,

N'il n'est si bon qu'ilz n'y treuvent a dire.

Leur faulz parler et leur male murmure

Empeschent gent, meismes l'air en corront,

Et qui plus ment volentiers plus en jure, 
C'est le droit cours que gent mesdisant vont [...]

(Cent balades, XCI, t. I, p. 91, vv. 12-18.)

\section{Christine à l'intention des uns et des autres, afin de les amener à vivre en paix.}

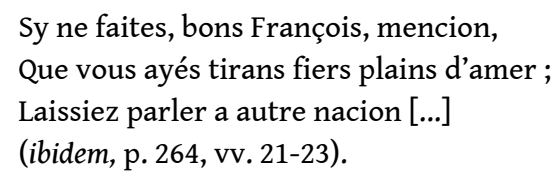
vrai dire qu'exceptionnellement dans l'œuvre lyrique. Mais même peu nombreux, ces exemples suffisent à confirmer l'extension universelle de la parole colportée telle que la représente Christine, au centre de la vie privée (mais s'agissant des princes, on peut douter qu'elle le soit vraiment), mais aussi, comme on peut le pressentir, de bien des aspects de la vie politique.

La perspective change sensiblement si l'on envisage les grandes écrits didactiques. Le thème de la parole multipliée, qu'elle soit rumeur menaçante ou bonne renommée, s'y déploie avec une richesse nouvelle, quoique le plus souvent à partir d'éléments déjà présents dans certaines des œuvres poétiques. Considérée ainsi dans tout son développement, la réflexion de Christine offre alors plusieurs traits fortement accentués.

19 Le premier d'entre eux est une implication personnelle beaucoup plus marquée de l'auteur. Parmi les interventions à la première personne ou les références autobiographiques assez nombreuses, qui sur ce sujet comme sur d'autres permettent à Christine d'installer au cœur de ses œuvres une représentation de son propre personnage, dont le public est curieux, nous n'examinerons que quelques exemples qui marquent un enrichissement du thème. Notons tout d'abord l'attitude réflexive qu'elle adopte dans le Livre de la mutacion de Fortune $e^{26}$ pour justifier son droit à la critique des mœurs, qu'elle distingue de la médisance ; car cette critique, ici appliquée aux nobles, tend au contraire, explique-t-elle, à combattre des vices qui alimentent parmi les étrangers des propos qui sont à la honte du royaume :

Si vous en vueil des maintiens dire,

Mais ne le fais pas pour mesdire 
De nullui, car qui autrui blasme

Sur soy meismes acquiert le blasme,

Mais les vices blasmer puis bien,

Qu'en aucuns je vi, et pou bien,

Dont me pesa, encore desplait,

Car aux estranges tenir plait

En ö̈, qu'aloient disant

Que le regne, qui reluisant

Souloit jadis estre en valour,

S'estoit tout tourné en folour

De nices meurs es nobles hommes [...]

(t. II, pp. 31-32, vv. 5143-5155).

Elle dira donc le vrai sur les gens de cour «qui n'ont envie/ Fors de mal faire», mais dont on n'ose faire justice. Ainsi la critique, mais aussi l'éloge, sont un devoir de l'écrivain moraliste: il témoigne personnellement de ce qu'il observe, ou perpétue le souvenir des hommes dont la renommée atteste la valeur :

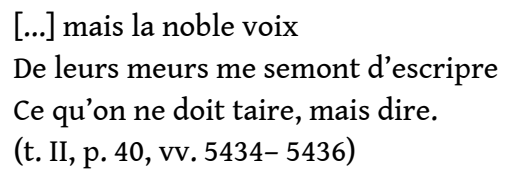

21 En s'attribuant cette fonction de distribuer le blâme et l'éloge, l'écrivain, selon le modèle qu'en propose Christine, en démontre la légitimité par une lucidité supérieure. Sa justification est en effet dans l'observation, l'analyse et le raisonnement, opposés à la confusion morale. C'est ainsi qu'elle caractérise celui qui «legierement gette son dart sur autrui par sa lengue », comme le font "tant de gens et de tous estaz si enclins a dire mal» (fol. $12^{v}$.) mais incapables de comprendre la vraie nature de la médisance :

[..... ] je me donne trop grant merveille de leur avuglement, car ilz ne regardent pas a leurs faiz et a ce que on peut dire d'eulx. Ains vraiement j'apperçoy que les plus bleciez d'aucun vice ou de pluseurs sont ceulx qui plus en blasment autrui ; et par ce cuident couvrir ce qui a l'ueil leur pent. Mais ilz se deçoivent, car ceulx qui leur oient dire, non obstant que pluseurs s'en rient et les y confortent et soustienent, sy s'en moquent ilz en derriere et dient que ilz sont gengleurs et mesdisans, et qu'ilz se eussent beau taire; et les en prisent moins et d'eulx se gardent, s'ilz font que sages. Sy ne scei veoir que telz gens gaignent a ainsi mesdire fors pechié et a eulx meismes diffame [...].(Prod'ommie de l'omme, fol. 12v).

Dans l'Advision, à propos des bruits qu'on a fait courir sur ses prétendues amours $^{27}$, Christine associe pareillement son propre témoignage à une explication rationnelle des rumeurs de ce genre :

[...] telz renommees viennent et sourdent communement, et souvent a tort, par grant acointance et frequentacions les personnes ensemble ou par conjectures et couleurs voirs semblables, [...] me suis mainte fois esmerveillee dont telz parolles sourdre pouoient, lesquelles estoient portees de bouche en bouche disans : «je l'ai ouy dire ». (III ${ }^{\mathrm{e}}$ partie, p. 104, 1l. 140-143, 150-152)

L'examen critique de ce qu'elle considère comme un des principaux vices du siècle. se trouve ainsi rehaussé par le recours à un tour personnel, à la fois vif et imagé. Ajoutons que la personnalisation du discours conforte d'une autre manière son autorité morale : on sait combien elle aime à donner une image en abyme du succès de ses propres ouvrages, évoquant «la voix» qui se répand parmi les princes au sujet de son travail et 
de ses livres, «en pou d'eure ventillez et [...] portez... en plusieurs pars et pays divers " (ibidem, p.111, 11. 16-17). Dispensatrice de bon et de mauvais "renom», la moraliste tient elle-même son autorité de la renommée qui s'attache à son œuvre, et pour une part de l'attestation qu'elle a soin d'en donner : elle se veut au centre d'une circulation de propos raisonnables et vrais, qu'elle tente de substituer au bruit détestable de la médisance.

Ce rôle imparti à l'écrivain n'est pas sans rapport avec un autre trait qui caractérise la représentation de la parole collective dans les œuvres d'enseignement: «la bonne renommée » qui n'est guère désignée que négativement dans la poésie sur les matières d'amour, y est considérée dans son existence propre, à plusieurs niveaux de la vie sociale. Sa réalité semble à vrai dire paradoxale ou contradictoire, car si elle est faite, tout comme la rumeur médisante, de paroles qui «volent» au loin, elle est cependant présentée comme un bien solide et durable. Pour la jeune princesse par exemple, elle "demourra plus longuement que quelconques aultre tresor " (Livre des trois Vertus, p. 41, 11. 20-21), conception qu'on rapprochera de l'usage judiciaire qui lui accorde la consistance d'un fait, souvent déterminant ${ }^{28}$. Chez les souverains, elle peut constituer un attribut permanent, comme il est dit de Charles V dont le «bon nom [...] peut acquerir degré de perpetuité $»^{29}$. Toutefois, dans cette perspective qui est politique, on observe que «la bonne renommée » réunit à la considération du temps présent, où s'exercent quotidiennement vertus et sagesse pratique, celle du temps historique de la tradition: Charles $\mathrm{V}$ est réputé sage dans ses moindres actions, mais aussi comme successeur "par espace de temps» de Clovis et Charlemagne. Car la «bonne renommée» en ce sens est une institution de la monarchie française. Et quand sa dimension actuelle fait défaut, il reste sa forme transcendante héritée du passé. Les princes, prêts à s'affronter dans la «honteuse bataille» de la guerre civile, peuvent-ils se rappeler l'esprit de concorde qui distinguait leur race et l'ensemble de leur «nacion », par une sorte de privilège français (en comparaison notamment des princes italiens)?

Et ne vous desplaise, où est à present le doulz sang naturel d'entre vous, lequel dès oncques seult estre le droit comble de la benignité du monde, de quoy tres les temps anciens sont raemplies toutes autentiques histoires, et de qui Fama seult corner ses chansons par tout l'universel monde? (Lamentacion sur les maux de la France, p. 180)

C'est avec cette gloire en danger de se perdre, mais inscrite dans une puissante tradition, que pourra renouer la reine Isabeau, si elle empêche les princes de s'affronter sur le champ de bataille: «en perpetuelle memoire de vous ramenteue, lui promet Christine, recommandée et louée es croniques et nobles gestes de France doublement couronnée de honneur seriez avec l'amour, graces, presens et humbles grans merciz de voz loyaulz subgiez » (Epistre à la reine, p. 255, 11. 49-53). ${ }^{30}$ Cette renommée historique est en fait constituée d'un ensemble de mythes qui entretiennent un rapport constant mais complexe avec l'écrit, bien au delà des chroniques officielles de la monarchie. Christine, après bien d'autres, dont son maitre Eustache Deschamps, y a vu un des lieux de travail principaux de l'écrivain, car les mythes de la monarchie doivent toujours être réactualisés et augmentés de figures nouvelles : c'est ainsi qu'elle conçoit son Charles $V$, et plus tard le Ditié de Jehanne d'Arc. ${ }^{31}$ La première partie de l'Advision-Cristine permet d'apprécier la nature exacte de ce travail. La France y est présentée d'emblée comme une figure prestigieuse, dont le «hault renom» qui retentit au loin a attiré à Paris 
Thomas de Pizan et sa famille. Pourtant une grande partie du discours placé dans la bouche du personnage de Libera, incarnation du royaume, va constituer une sorte de démonstration, sous forme d'histoire allégorique, de cette gloire si clairement posée au départ: tout le détail d'une brillante tradition, avec ses aléas, sera longuement repris, comme si les malheurs du temps obligeaient à cette réélaboration d'un bien pourtant acquis de longue date. C'est que la renommée où se résument toutes les qualités du royaume et de ses princes est menacée par une parole plus volatile, mais destructrice, cette «famme villaine » (Advision, $1^{\mathrm{e}}$ partie, p. $\left.37,1.8\right)$ qui naît du désordre intérieur, et bientôt de la guerre civile. Dans cette lutte entre le passé prodigieux et le présent infâme, cette renommée héritée, qu'on peut dire avant la lettre nationale, doit être mobilisée par le travail d'écriture, lui aussi multiplicateur de paroles.

En intervenant sur les différentes scènes où se déploie la parole colportée, Christine a tenté un effort de clarification rationnelle dont nous avons déjà rencontré plusieurs exemples. C'est là une constante de son travail dans ce domaine : il tient tout à la fois de la théorie morale, de l'observation des mœurs et de la sagesse pratique. Il suffira de caractériser la démarche méthodique qu'elle adopte, sans prétendre rendre compte d'un corps de réflexion qui d'une œuvre à l'autre se développe avec cohérence.

Il est significatif qu'au moment d'entamer l'exposé des vertus du "sage roy Charles V ", Christine propose une définition analytique de la première d'entre elles, la «noblece de courage », et présente immédiatement «le bien de renommée» (Charles V, I, pp.10-11) comme réunissant en lui les trois dispositions morales - goût des choses élevées, amour de la vertu, sagesse dans la conduite des affaires - qui constituent cette éminente qualité. Car elle n'est autre que leur forme visible, le «digne corps imaginable et non palpable» (ibidem, p.11) sous lequel elles s'offrent au regard. Cette visibilité des grandes qualités morales est donc tout à fait essentielle. Or c'est précisément ce couronnement nécessaire des vertus que les médisants tendent à détruire dans les cours, souvent avec l'agrément des grands, comme Christine l'a plusieurs fois noté, écrivant par exemple dans la Prod'ommie de l'omme:

[...] est grant merveille comment pluseurs grans seigneurs et autres riches et puissans hommes prennent en teles gens plaisir [...] Et pleust a Dieu que les princes les haïssent autant qu'ilz font les traitres,-et sy ne valent ilz gairez moins. Mais aucuns dient qu'ilz les y soustiennent et portent, et pour ce ilz se enhardissent. (Prod'ommie de l'omme, fol.12 ${ }^{\mathrm{v}}-13^{\mathrm{r}}$ )

C'est à partir de ce constat que Christine entreprend une critique théorique de la médisance, qu'elle associe dans le Livre des trois Vertus à un recensement méthodique des dispositions qui doivent en arrêter les effets. Sur le plan moral, ce vice que d'aucuns tiennent pour négligeable tant il est coutumier, est placé dans une position symétrique de celle qu'elle attribue à la bonne renommée, c'est-à-dire très haut dans l'échelle du mal :

[...] ainsi en diffamant autrui, soit par mauvaise coustume ou autrement, quoi que pluseurs pou en facent conte, sont consummez en un seul coup pluseurs pechiez mortelz. (Prod'ommie de l'omme, fol. $12^{\text {r }}$ )

Souvent inspirée par la haine et l'envie, la médisance incite en outre les autres à agir de même et se révèle une source de corruption morale très répandue. Lorsque Christine, dans le Livre des trois Vertus, entreprend d'analyser plus finement ses ressorts dans la pratique ordinaire de la vie de cour, elle mentionne, parmi ses mobiles, la simple «opinion », c'est-à-dire 
les jugements prononcés sans connaissances certaines, à partir d'apparences souvent interprétées à tort ${ }^{32}$. C'est sur ce point qu'elle concentre particulièrement son attention : les propos qui trouvent là leur origine sont les plus fréquents, selon les mœurs de la cour, et parmi les plus dangereux, parce qu'apparemment désintéressés et donc plus crédibles. Ce sont eux qui peuvent causer les plus grands désordres, jusqu'à une forme de subversion, par une prolifération de discours qui échappent à tout contrôle. L'auteur les montre s'augmentant irrésistiblement d'éléments nouveaux, esquissant ainsi une théorie de la rumeur qu'alimentent les stéréotypes propres à un milieu.

Il est d'autres aspects plus diffus de la réflexion théorique de Christine sur la médisance: ils concernent notamment les rapports entre les différentes formes de «mauvais langage » qui contribuent à grossir le flot de la parole collective. En effet, si la médisance en est la source principale, Christine signale fréquemment que d'autres vices très communs lui sont associés. Ainsi quand de jeunes chevaliers médisent des femmes, comme on l'a vu dans plusieurs scènes et portraits, ou que des dames de cour sont à l'origine de rumeurs discréditant leur maîtresse, le mensonge, le bavardage, la jactance, la moquerie sont généralement de la partie. Il est probable que cette vision à la fois large et analytique du phénomène se rattache indirectement à une longue tradition théologique de définition systématique des «péchés de la langue » qui avait d'abord concerné la vie monastique, puis les activités pastorales ${ }^{33}$ : Gerson, dont Christine, comme on sait, était proche, en était un des continuateurs par certains de ses écrits ${ }^{34}$. Sur quelques points précis, la convergence est nette entre la problématique théorisée dans ce domaine et la version laïque qu'en propose Christine : par exemple quand elle se demande si elle peut blâmer les vicieux sans se rendre coupable de " diffame " - la distinction entre diffamation et correction constituait depuis longtemps un problème classique ${ }^{35}$-, ou encore quand elle compare la gravité de la flatterie et de la médisance, et dénonce la duplicité des flatteurs de cour, qui souvent s'empressent de médire de ceux qu'ils ont encensés: la complicité secrète entre «adulatio» et "detractio», péchés apparemment opposés, avait été bien caractérisée par les théologiens ${ }^{36}$. Sans doute faut-il songer à éclairer parfois par ce contexte originel la valeur qu'elle donne à certains mots, comme "murmure», qui avait désigné un des péchés monastiques les plus graves ${ }^{37}$, une sourde révolte contre les charges imposées par les supérieurs, ou certaines images, comme celle de l'abbaye que nous rencontrerons à propos de l'entourage d'une princesse. Plus généralement, à l'arrière plan des représentations très concrètes que propose Christine pour illustrer les effets les plus nocifs de la parole collective, il faut deviner la haute science dont l'apologie conclut son Advision, Philosophie « en fourme de Sainte Theologie $»^{38}$.

31 Cependant le propos de Christine est essentiellement pratique, voire pragmatique, et vise en premier lieu la cour des princes, qui dans le Livre de la paix comme dans le Livre des trois Vertus apparaît, au moins implicitement par les conseils prodigués, comme un lieu où fermentent la médisance, le mensonge et la rumeur : la maîtrise des paroles qui circulent y est aussi nécessaire que difficile. Aussi la définition des qualités de l'officier de cour, du serviteur dévoué aux intérêts d'un prince, est-elle, comme celle des dames de la cour, en grande partie négative : il aura "bonne bouche", s'abstenant de faire des rapports sur autrui, et se montrant prompt à apaiser les différends que d'autres 
s'emploient à faire naître. En résumé, il sera tout l'opposé de celui qui suscite le désordre par ses discours :

[... ] l'omme rioteux, noiseux et discordant puet esconmouvoir, non seulement la court d'un prince, mais tout un pays ou une ville par semer ses mauvaises parolles (Livre de la paix, p. 87)

Concernant les dames de cour, Christine avait de même exposé dans le Livre des trois Vertus, avec beaucoup de minutie, cette sagesse de précaution esquissée dans d'autres ouvrages: les grandes dames doivent à la fois observer la plus grande prudence dans leurs relations, pour éviter de provoquer dépits et rancunes qui seraient à l'origine de propos néfastes, et contrôler strictement gestes, attitudes et paroles que la médisance pourrait exploiter. Elles doivent aussi savoir gouverner leur entourage avec fermeté, isolant comme «mauvaise berbiz [...] roingneuse» (p. 144, 1. 42) toute bavarde trop prompte à médire, et contrôlant les relations avec l'extérieur, comme dans «une abbaye bien ordonnee", dont les moines sont tenus au secret (p. 147, 1. 24). Mais l'instruction que Christine donne aux princesses va bien au delà de ces gestes défensifs : elle constitue une véritable politique de la renommée, sous la forme d'un pharisianisme systématique. Il s'agit d'obtenir des propos favorables de tous les états de la société, par des bienfaits et des égards ostensibles qui s'adresseront aux gens d'église, "pour avoir renommee par eulx» (p.67, 1. 51), mais aussi aux chevaliers, aux clercs et aux bourgeois, sans oublier les "petites femmes de village", dont on saura orienter le «caquet». Il n'est pas de milieu dont les propos ne doivent être suscités par des gestes habiles de la princesse, car Christine souhaite que «leurs voix et leurs paroles lui puissent estre, se mestier est, escu et deffense contre les murmures et les rapors de ses envieux mesdisans, et les puissent estaindre» (p.66, 11.19-21). Ainsi sera maitrisé l'ensemble des discours collectifs qui circulent dans la société, ensemble sans doute très différent de ce que nous appelons opinion publique - car ces discours se forment séparément et n'ont pas d'organe commun -, mais néanmoins haussé au niveau d'une sorte d'entité politique.

Comme il apparaît assez clairement par ces derniers exemples, Christine privilégie généralement dans ses traités une perspective qui fait du contrôle de la parole publique un attribut du pouvoir: c'est aux princes à empêcher qu'elle ne s'empare de signes qu'elle traduira de manière fausse et néfaste à mesure qu'elle en amplifiera l'écho ; c'est à eux également de lui fournir de quoi alimenter des discours favorables dont la diffusion contribuera à asseoir leur puissance. Si l'entourage des princes est déjà un lieu où la parole colportée prend aisément une dimension politique, il convient de considérer des scènes plus vastes, à l'échelle du royaume et même de l'Europe, pour en comprendre tous les enjeux.

Cet élargissement de la perspective, qui semble de plus en plus marqué dans les œuvres de Christine à partir de son Charles $V$, revêt cependant des formes assez différentes, en partie déterminées par les événements. Son portrait historique du «sage roy» est organisé pour traduire les effets politiques des qualités personnelles et privées du souverain. De ce point de vue, un des traits les plus significatifs est sans doute que Charles change radicalement de mœurs en montant sur le trône. Christine met en valeur cette réforme, qu'on ne peut soupçonner de «simulacion soubz vesteure fainte ", parce qu'elle est inspirée par une claire compréhension de la «faame deue à digneté et trosne royal» (Charles V, I, p. 37): en l'espace de trois ans, «le renom porté de ses vertus en la presence de pluseurs princes et terres estranges comme de prince 
solempnel en toutes graces» devient un instrument de sa politique et lui vaut le ralliement de plusieurs de ses ennemis (ibidem, p. 125). Bien des dispositions adoptées par le roi, comme l'ordonnance somptueuse de "son chevauchier", ont une portée analogue ; mais Christine insiste en outre très significativement sur le sens qui en sera transmis aux «successeurs à venir» (p. 51), car le roi conçoit politiquement son propre renom, non pas seulement dans ses effets immédiats, mais à l'échelle d'une tradition monarchique. Sous un autre langage, le point de vue n'est guère différent quand aux pires moments de la guerre civile, Christine évoque, comme nous l'avons vu, l'honneur traditionnel de la France, ainsi interpelée :

Car ne seras-tu pas acomparée de cy en avant aus estranges nacions, là où les freres germains, cousins et parens par faulse envie et convoitise s'entreocient comme chien? (Lamentacion sur les maux de la France, p. 182).

Qu'en des circonstances aussi tragiques, ce qu'en dira-t-on européen puisse être un des principaux arguments avancés pour soutenir l'appel en faveur de la paix intérieure marque très significativement la place accordée au renom du royaume, qui se confond avec la réputation de ses princes: il constitue un des fondements de son existence même.

Dans ses derniers traités, Christine dénonce une autre forme particulièrement néfaste à ses yeux de la parole collective: celle qui échappant au contrôle du souverain se développe dans le peuple, soit pour alimenter des dissensions entre les différents " estas» du royaume, soit pour critiquer les princes Cette préoccupation, déjà très marquée dans le Livre du corps de policie (1406-1407), se manifeste avec plus d'insistance encore dans le Livre de la paix (1413). C'est à l'évidence un effet du poids croissant du «commun» de Paris à la veille de la révolution cabochienne ${ }^{39}$. Sur ce terrain particulier, le thème conserve plusieurs de ses traits fondamentaux, mais sa configuration se modifie, et le lexique évolue. Déjà dans la Prod'ommie de l'omme, à propos de l'usage du mensonge dans toutes les classes de la société, Christine avait relevé la propension du peuple à médire du caractère et des mœurs des seigneurs et des dames. Quand cette "médisance» prend un tour nettement politique - il s'agit désormais de ceux qui médisent «d'ordonnances de princes» (Livre du corps de policie, p. 101, 1. 9) -, la condamnation se fait plus brutale : en pareille matière, seul le silence convient au peuple, dont la parole est essentiellement mensongère et dangereuse. Aussi « les sages doivent amonnester les simples et les ignorans de eulx taire de ce de quoy ne leur appertient à parler, et dont grant peril peut venir et nul preu ». (ibidem, p. 101, 11 . 21-23). Christine reste donc attentive aux moyens d'endiguer cette menace: «les sages bourgois ", agissant comme intermédiaires, tenteront de désamorcer les causes de mécontentement et d'apaiser «les murmuracions du peuple» (p. 100, 1. 36). Mais dans les deux traités où le problème est longuement évoqué, la perspective adoptée tient moins de la sagesse pratique - qui inspire par exemple les minutieuses instructions du Livre des trois Vertus - que d'une rigoureuse position de principe à l'égard du peuple, qui de tout temps a constitué une menace de subversion: il ne sera guère question de contrôler sa parole, mais seulement de la proscrire, et cette condamnation, qui exclut toute concession, sera logiquement appuyée de nombreux «exempla» empruntés à la bible et à l'histoire ancienne. À cette attitude péremptoire répond d'ailleurs une perception du phénomène différente de ce que l'on a pu observer au sujet des autres formes de médisance. Il n'est plus guère question en effet de la propagation et de la multiplication des propos dangereux, car telle qu'elle est représentée, la parole 
populaire est une, massive, bruyante et d'emblée redoutable. Par son existence même, elle est séditieuse, car toujours dirigée contre les chefs légitimes de la société, ce que traduisent les termes «murmure », «murmurer », et parfois «rumeur ». Dans le Livre de la paix, Christine, s'adressant au "peuple universal, de toutes l[é] parties du monde » qui aurait la folie de se «mouvoir à rebellion » (p. 128), lui rappelle solennellement que «desplaist a Dieu murmure de subgiez vers prince et contre leurs mageurs » (p. 129); et plus loin dans le même ouvrage, «rumeurs » désigne les «mauvais esplois » des fureurs populaires (p. 132): plus qu'en tout autre contexte, la parole collective est identifiée à une action essentiellement destructrice.

L'extraordinaire extension que prend le thème de la parole multipliée, depuis les première ballades jusqu'aux derniers traités écrits dans des temps calamiteux, sa très grande variété, pourraient suggérer qu'il ne comporte ni unité ni cohérence. Nous avons cru possible de montrer au contraire qu'entre les différentes formes qu'il revêt, il existe de multiples passages: par exemple de la poésie amoureuse à la critique des mœurs et à la politique, de la défense des femmes calomniées à l'instruction des princes. D'autre part, certaines affirmations fondamentales se font jour sur ce terrain : l'extrême danger, moral aussi bien que politique, de la parole divaguante, ennemie de l'ordre et corruptrice, à quoi s'opposent, rapprochés sur ce point comme sur d'autres, le prince et l'écrivain, tous deux à l'origine de discours bénéfiques dont l'effet s'étend au loin.

Cependant nous avons également discerné, en suivant l'enrichissement de ce thème tout au long de l'œuvre de Christine, une dualité de points de vue très caractéristique de sa manière : l'attention portée aux traits les plus précis, les plus concrets de la vie sociale s'y trouve associée à un effort et à une compétence théoriques très affirmés. Dans certains de ses meilleurs moments, et tout particulièrement sur le sujet de la parole auquel elle a toujours porté un grand intérêt, Christine est une théoricienne de la chose vue. Cette dualité de ses moyens d'investigation peut contribuer à expliquer que sa réflexion ait pu s'adapter et rester assez productive pour répondre aux changements d'une époque tourmentée.

\section{NOTES}

1.Je remercie très vivement Thelma Fenster de m'avoir permis de consulter avant parution son excellent article, «La fama, la femme, et la Dame de la Tour : Christine de Pizan et la médisance.", à paraître dans Au Champ des écritures (Actes du III Colloque international sur Christine de Pizan, Lausanne, 18-22 juillet 1998), publié par Eric Hicks, avec la collaboration de Diego Gonzalez et Philippe Simon, comité scientifique : Eric Hicks, Jean-Claude Mühlethaler, Alain Corbellari, Catherine Müller.

2. Voir Emmanuèle Baumgartner, "Trouvères et "losengiers" ", Cahiers de Civilisation Médiévale, $X^{e}-X I I^{e}$ siècles, Poitiers, Centre d'Études Supérieures de Civilisation Médiévale, 1982, n 3-4, pp. 171-178; Marcel Faure, «Le Losengier dans la chanson des trouvères des XII et XIII siècles", Félonie, Trahison, Reniements au Moyen Âge, Actes du troisième colloque 
international de Montpellier, Université Paul-Valéry (24-26 novembre 1995), Montpellier, Publications de l'Université Paul-Valéry, Les Cahiers du C.R.I.S.I.M.A., n 3, 1997, pp. 189-195; Erich Köhler, «Les Troubadours et la jalousie » Mélanges de Langue et de Littérature du Moyen Âge et de la Renaissanse offerts à Jean Frappier, Genève, Droz, 1970, pp. 543-559; Henri Rey-Flaud, La névrose courtoise, Paris, Navarin, 1983, pp. 9-35. 3. L'image est fréquente, aussi bien dans la poésie amoureuse que dans la littérature politique, où le fauteur de désordre et de discorde est accusé de «semer ses mauvaises parolles » (Le Livre de la paix, éd. Charity Cannon Willard, 'S-Gravenhage, Mouton, 1958, p. 87).

4. Le Livre de l'Advision Cristine, éd. critique par Christine Reno et Liliane Dulac, Paris, Champion ("Études Christiniennes»), sous presse (ci-dessous Advision).

5. Citons par exemple ce rondeau : «Pour quoy me doit on blasmer/De mon doulz ami amer? [...] En fault il tel plait semer/Partout pour moy diffamer? » (XV, vv. 2-3 et 9-10, Euvres poétiques de Christine de Pisan, éd. Maurice Roy, 3 vol., Paris, Didot («Société des Anciens Textes Français »), 1886-1896 (ci-dessous éd. M. Roy), t. I, p. 156. On trouvera plus loin ces termes employés dans divers contextes.

6. Christine écrit par exemple, à propos de sa réputation naissante : «[...] la voix courust ja, et meismes entre les princes, de l'ordre et maniere de mon vivre, c'est assavoir a l'estude ", Advision, III partie, p. 111, 11. 8-9.

7. L'Epistre Othea, éd. Gabriella Parussa, thèse de doctorat, Université de Paris VII, 1995, à paraître chez Droz, Allégorie XLII : «Et si est deffendu, ce dit saint Augustin, toute faulse accusacion, murmuracion, detraccion, tout faulx rapport et diffamacion d'autrui ».

8. Le Livre de la Prod'ommie de l'homme (ou Le Livre de Prudence), éd. en préparation d'Eric Hicks et de Simone Pagot, dans la copie obligeamment communiquée par Eric Hicks, (d'après le manuscrit Biblioteca Apostolica Vaticana Reg. lat. 1238, fol. 1-46) : « Ne croiz pas de legier rumeurs, crimes ne souspeçons » (fol. $\left.28^{\mathrm{r}}\right)$.

9. Le Livre de la Cité des dames, dans La Città delle Donne, a cura di Patrizia Caraffi, Edizione di Earl Jeffrey Richards, Milan, Luni Editrice, 1998 (seconde édition), p. 372. 10. Cent balades, LVIII, v. 19, éd. M. Roy, t. I, p. 59.

11. Sur ces termes, voir Claude Gauvard, «Rumeurs et stéréotypes à la fin du Moyen Âge ", La circulation des nouvelles au Moyen Âge, XXIV ${ }^{e}$ Congrès de la S. H. M. E. S. (Avignon, Juin 1993), Paris, Publications de la Sorbonne,1994, pp. 157-177, notamment p. 169.

12. À propos de cette question, nous avons consulté principalement : Rosalind BrownGrant, Rhetoric and Authority: Christine de Pizan and the moral Defence of Women : Reading Beyond Gender, Cambridge, Cambridge University Press, 1999 (particulièrement pp. 193-206, "politics of visibility »); Charles Brucker, «Élévation, gloire et renommée dans quelques œuvres de Christine de Pizan ", Sur le chemin de longue étude... : Actes du colloque d'Orléans, juillet 1995, éd. Bernard Ribémont (Études Christiniennes), Paris, Champion, 1998. pp. 45-64 ; Carla Casagrande, Silvana Vecchio, Les Péchés de la Langue; Discipline et éthique de la parole dans la culture médiévale, Paris, Cerf, 1991 ; Andrée Courtemanche, "La rumeur de Manosque : femmes et honneur au XIV ${ }^{e}$ siècle ", Normes et pouvoir à la fin du moyen âge, Actes du colloque "La recherche en études médiévales au Québec et en Ontario "16-17 mai 1989, publiés par Marie-Claude Déprez-Masson, Montréal, Ceres, Éd. Inedita et Rara 7, 1990, pp. 127-144; Claude Gauvard, «La fama, une parole 
fondatrice », Médiévales, n²4-printemps 1993, «La Renommée», pp. 5-13; Claude Gauvard, «Christine de Pizan et ses contemporains : L'engagement politique des écrivains dans le royaume de France aux XIV et XV siècles ", Une femme de Lettres au Moyen Âge. Éudes autour de Christine de Pizan, articles réunis par Liliane Dulac et Bernard Ribémont, Orléans, Paradigme, 1995, pp. 105-128; Nicole Pons, « De la renommée du royaume à l'honneur de la France ", Médiévales, op. cit., pp. 101-116 ; Danielle RégnierBohler, «Voix littéraires, Voix Mystiques », Histoire des femmes en occident, Le Moyen Äge, sous la direction de Georges Duby, Michelle Perrot, Paris, Plon, 1990, pp. 443-500 ; Gilles Roques, «La Réputation dans la langue française médievale : Glossaire onomasiologique du Moyen Français ", Médiévales, op. cit., pp. 45-56.

13. Cuvres poétiques de Christine de Pisan, éd. Maurice Roy, op. cit.

14. Le Livre des Epistres sur le Roman de la Rose, éd. Eric Hicks, Paris, Champion («Bibliothèque du XV siècle » XXLIII), 1977, réimpr. 1996, p. 146, 1. 1004-1005.

15. Éd. M. Roy, t. I, p. 209.

16. Sur la rumeur en général, voir : Ruth Amossy, Anne Herschberg Perrot, Stéréotypes et Clichés, langue, discours, société, Paris, Nathan, 1997 ; Jean-Noël Kapferer, Rumeurs. Le plus vieux média du monde, Paris, Seuil,1990.

17. Éd. M. Roy, t. III, pp. 296-297 ; Cent ballades d'amant et de dame, éd. Jacqueline Cerquiglini, Paris, U. G. E. ( 10/18»), 1982, p. 120. Voir : Christine de Pizan and French Lyric, ed. E. J. Richards, Gainesville, University of Florida, 1998 ; Charity Cannon Willard, "Cent ballades d'amant et de dame, Criticism of courtly love", Court and Poet: Selected Proceedings of the Third Congress of the International Courtly Literature Literature Society, ed. Glyn S. Burgess, Liverpool, Cairns, 1981, pp. 357-364 ; Liliane Dulac, « Dissymétrie et échec de la communication dans les Cent ballades d'amant et de dame de Christine de Pizan ", Lengas, 22, 1987, pp. 133-146.

18. Éd. M. Roy, t. I, pp. 226-227.

19. Ibidem, t. I, p. 114.

20. Éd. M. Roy, t. II, p. 5. Voir Lori Walters, «The Woman Writer and Literay History : Christine de Pizan's Redefinition of the Poetic Translation in the Epistre au dieu d'amours ", French Literature Series 16, 1989, pp. 1-16.

21. Éd. M. Roy, t. II, p. 18 ; voir Helen Solterer, «Christine’s Way. The querelle du Roman de la rose and the Ethics of a Political Response ", The Master and Minerva, Disputing Women in French Medieval Culture, Berkeley, Los Angeles, London, University of California Press, 1995, pp. 151-175, et Woman Defamed and Woman Defended, An Anthology of Medieval Texts, ed. Alcuin Blamires with Karen Pratt and C. W. Marx, Oxford, Clarendon Press, 1992, ch. 9, «A woman defends women. Christine de Pizan », pp. 278-302.

22. Le Livre du Duc des vrais amans, éd. M. Roy, III, p. 59-208. Nous nous référons à cette édition, mais nous consultons également celle plus récente de Thelma S. Fenster, Binghamton, NY («Medieval and Renaissance Texts and Studies», 124), 1994. Voir aussi : The Book of the Duke of True Lovers, translated with an introduction by Thelma S. Fenster, with lyric poetry translated by Nadia Margolis, New York, Persea Books, 1991 ; Liliane Dulac, "Christine de Pizan et le malheur des vrais amans ", Mélanges de littérature médiévale offerts à Pierre Le Gentil, éd. Jean Dufournet et Daniel Poirion, Paris, SEDES, 1973, pp. 222-233 ; Judith Laird and Earl Jeffrey Richards, "Tous parlent par une mesmes bouche: Lyrical Outbursts, Prosaic Remedies, and Voice in Christine de Pizan ", Christine de Pizan and French Lyric, op. cit., pp. 103-131. Christine McWebb, « Lyrical Conventions 
and The Creation Female of Subjectivity in Christine de Pizan's Cent ballades d'amant et de dame ", Christine de Pizan and Medieval French Lyric, op. cit., pp. 168-183. Barbara K. Altmann, The Love Debate Poems of Christine de Pizan. Le livre du Debat de deux amans. Le livre des Trois jugemens. Le livre du Dit de Poissy, Gainesville, University Press of Florida, 1998.

23. Le Livre des trois Vertus, éd. Charity Cannon Willard et Eric Hicks, Paris, Champion, 1986 ; voir : Liliane Dulac, «The Representation and Functions of feminine Speech in Christine de Pizan's Livre des Trois Vertus» (translated by Christine Reno), Reinterpreting Christine de Pizan, ed. Earl Jeffrey Richards, with Joan Williamson, Nadia Margolis and Christine Reno, University of Georgia Press, Athens and London, 1992.

24. Jean Gerson, Traité des diverses tentations de l'ennemi, Euvres complètes, éd. Mgr Glorieux, Paris, Tournai, Rome, New York, 1960-1973, 10 vol., t. 7, p. 351.

25. Le Livre du corps de policie, éd. Angus J. Kennedy, Paris, Champion, («Études Christiniennes»), 1998.

26. Le Livre de la mutacion de Fortune, éd. Suzanne Solente, 4 vol., Paris, Picard, 1959-1966.

27. Gianni Mombello, «J.-M.-L. Coupé e H. Walpole : Gli amori di Christine de Pizan », Studi Francesi, 16, 1972, pp. 5-25.

28. Voir Cl. Gauvard, « Rumeurs et stéréotypes...», art. cit., pp. 167-169.

29. Le Livre des fais et bonnes meurs du sage roy CharlesV, éd. Suzanne Solente, 2 vol., Paris, Champion, 1936-1940, t. I, p. 10 ; voir aussi la traduction d'Eric Hicks et Thérèse Moreau, Le Livre des Faits et Bonnes Mœurs du roi Charles V le Sage, Paris, Stock, 1997.

30. Epistre à la reine, éd. Angus Kennedy, Revue des Langues Romanes, Christine de Pizan, études recueillies par Liliane Dulac et Jean Dufournet, 1988, 2, pp. 253-264. Rosalind Brown-Grant, «Les exilées du pouvoir? Christine de Pizan et la femme devant la crise du Moyen Âge finissant ", Apogée et déclin, textes réunis par Claude Thomasset et Michel Zink, Paris, Presses de l'Université de Paris-Sorbonne, 1993, pp. 211-223. Eric Hicks, «Une femme dans le monde: Christine de Pizan et l'écriture de la politique », L'Hostellerie de Pensée, études sur l'art littéraire au Moyen Âge offertes à Daniel Poirion par ses anciens élèves, textes réunis par Michel Zink et Danielle Bohler, publiés par Eric Hicks et Manuela Python, Presses de l'Université de Paris-Sorbonne, 1995, pp. 233-243.

31. Le Ditié de Jehanne d'Arc, éd. et tr. Angus J. Kennedy et Kenneth Varty, Oxford, Society for the Study of Medieval Languages and Literature, 1977.

32. Livre des trois Vertus, p. 141 et 143-146.

33. Voir notamment, dans Les péchés de la langue, les chapitres concernant «mendacium », « detractio », « iactantia », « derisio », « multiloquium ».

34. Gerson, Pour qu'on réfrène sa langue, Euvres complètes, t. 7, pp. 400-404; voir Les péchés de la langue, p. 170, note 1.

35. Les péchés de la langue, p. 242-243.

36. Ibidem, p. 244.

37. Voir le chapitre consacré au «murmur» dans Les péchés de la langue, pp. 181-186: notons que l'exemplum qui illustre traditionnellement l'extrême gravité de cette faute dans la vie monastique, le murmure des Hébreux au désert contre Moïse et leur châtiment par Dieu, est utilisé par Christine pour condamner la révolte du peuple contre les princes (Livre de la paix, pp. 128-133, « comment desplait a Dieu rebellion et murmure de commun vers seigneurs»). 
38. Advision, III $^{\mathrm{e}}$ partie, p. 140, l. 12.

39. Françoise Autrand, Charles VI, La folie d'un roi, Paris, Fayard, 1986.

\section{AUTEUR}

LILIANE DULAC

Université de Montpellier III 